\title{
Agregados Monetarios, Inflación y Actividad Económica en México
}

\author{
Daniel G. Garcés Díaz \\ dgarces@banxico.org.mx
}

Abril de 2002

Documento de Investigación No. 2002-07

Dirección General de Investigación Económica

BANCO DE MÉXICO

\footnotetext{
`Agradezco a Adalberto González, Rafael Gómez-Tagle, Alejandro Werner y los participantes en seminarios en el Banco de México por sus útiles comentarios y sugerencias. Rocío Elizondo colaboró de modo eficiente en todos los aspectos de este documento. El autor es por supuesto responsable de cualquier error u omisión. Asimismo, el contenido de este trabajo no refleja necesariamente las opiniones del Banco de México.
} 


\title{
Agregados Monetarios, Inflación y Actividad Económica en México
}

\author{
Daniel G. Garcés Díaz
}

Abril de 2002

Documento de Investigación No.2002-07

\section{RESUMEN}

El objetivo de esta investigación es analizar la relación de los distintos agregados monetarios con la inflación y la actividad económica en México desde 1980. Primero se examinan las propiedades de estabilidad y exogeneidad de las demandas de los distintos agregados. El método de estimación utilizado permite probar directamente las propiedades de estabilidad de cada relación de largo plazo. Las demandas más estables son las de billetes y monedas y de M2. Las demandas de M1 y M4 muestran posible inestabilidad de diferente origen. A continuación, se cuantifica el contenido informativo de dichos agregados para la predicción de la inflación y de la actividad económica. Los resultados indican que la utilidad de los agregados monetarios como indicadores adelantados de la inflación y de la actividad económica es, en el mejor de los casos, modesta. También puede concluirse que debido a las propiedades de mayor estabilidad, poder predictivo y contenido informativo, la definición más estrecha de dinero es preferible sobre agregados más amplios para analizar la relación en México del dinero con otras variables macroeconómicas. 


\section{Introducción}

El enfoque monetarista ha considerado a la cantidad de dinero como un aspecto crucial para la determinación de los principales agregados macroeconómicos. Uno de los problemas empíricos más conocidos en este campo es el de la estimación de una demanda de dinero, es decir, de una función que explique el nivel de los saldos monetarios deseados por los agentes económicos con base en unas cuantas variables relevantes. Esta relación es útil para los bancos centrales a fin de poder programar la emisión de circulante y como parte integral del análisis macroeconómico. ${ }^{1}$

La relación entre el dinero y otras variables debe ser estimada de modo preciso y robusto para que sea de utilidad. A tal fin, es importante que los parámetros estimados sean constantes y que la ecuación ajuste bien los datos y genere pronósticos eficientes durante el período relevante (es decir, la demanda de dinero debe ser estable). Otro aspecto importante a considerar es el de la exogeneidad de las variables. Ello consiste en la determinación de cuáles factores pueden tomarse como dados para fines de estimar y hacer pronósticos con dicha ecuación. Así, este documento responde a dos objetivos. El primero es examinar las propiedades de estabilidad y exogeneidad de las demanda de los distintos agregados monetarios. El segundo consiste en evaluar el contenido informativo de dichos agregados para la predicción de la inflación y de la actividad económica.

El primer objetivo se discute en el contexto de los modelos de demanda de dinero para cada agregado. En varios artículos sobre este tema escritos para el caso de México $^{2}$ se afirma que dicha función es estable a pesar de los muchos cambios habidos en el entorno económico. Sin embargo, la "demostración"' de dicha propiedad que se presenta en tales trabajos no puede considerarse como definitiva. Ello, debido a que las pruebas estadísticas ahí utilizadas para tal fin se aplican al modelo de corto plazo (es decir, al proceso de ajuste) y no a la relación de largo plazo, que es la que se enfatiza $^{3}$ en la teoría económica .

Lo anterior no es una simple cuestión académica. Analizar la estabilidad de la demanda de dinero de largo plazo es crucial para cualquier programa monetario en horizontes mayores que unos cuantos meses. En el caso de México hay razones para estar atento a cualquier cambio en dicha

\footnotetext{
${ }^{1}$ En la actualidad el énfasis en el estudio de la demanda de dinero es menor que en el pasado. Ello debido a la inestabilidad que la relación entre precios y dinero presenta.

${ }^{2}$ Ver, por ejemplo, Khamis y Leone (1999) y Cuthberson y Galindo (1999).

${ }^{3}$ Generalmente, la teoría económica específica una forma funcional (como la ecuación 1) que debe cumplirse en el equilibrio de largo plazo; sin embargo, la teoría no especifica, por ejemplo, cuántos rezagos de las variables debe tener una ecuación de ajuste. No obstante, en el caso de la demanda de dinero, hay algunas restricciones que deben cumplirse para que una ecuación de corto plazo pueda ser interpretada como una demanda de dinero. Estas restricciones se señalan en la sección 5.
} 
relación. Recientemente Khamis y Leone (1999) demostraron que es posible encontrar un mecanismo de corrección de error estable para la demanda de billetes y monedas aún durante períodos de turbulencia.

Sin embargo, lo anterior no es suficiente. La razón es que se pueden esgrimir ejemplos recientes donde se demuestra que ciertos eventos económicos pueden cambiar los parámetros de largo plazo, independientemente de lo que ocurra con el mecanismo de corrección de error. Vega (2000) encontró que los parámetros de la demanda de dinero amplio (ALP2) en España cambiaron a raíz de la apertura financiera. Gabriel et al. (1999) también descubrieron que la relación de largo plazo en Portugal sufrió un cambio estructural.

En este trabajo se estiman robustamente los parámetros de largo plazo de la demanda real de dinero para cada agregado como función de la tasa de interés y de la actividad económica. En el ejercicio se obtuvieron, tal y como la teoría lo postula, elasticidades ingreso razonables y un signo negativo para la semi-elasticidad de la tasa de interés en todos los casos. La estabilidad de estas relaciones de largo plazo fue sometida a prueba directamente (a diferencia de lo que se encuentra en la literatura previa) y se derivaron modelos de corrección de error parsimoniosos que también tienen buenas propiedades estadísticas. El análisis de estos modelos establece que las ecuaciones de demanda de dinero no son invertibles. Este resultado es indicativo de que en México el dinero es determinado por los precios y no a la inversa.

El segundo objetivo se llevó a cabo suponiendo que los movimientos de los agregados monetarios son resultado de las decisiones del banco central. En este enfoque, el dinero ayuda a explicar y a predecir otras variables de importancia (precios, producto y tasa de interés). Para la inflación se utiliza el enfoque sugerido por el modelo $P^{*}$, aunque no en todos los agregados es posible aplicar una versión estricta del mismo. Los resultados indican que las funciones de los agregados monetarios tienen un poder predictivo apenas modesto para la inflación. Por tanto, dichos agregados son dominados por otro tipo de modelos que incluyen a otras variables (notablemente el tipo de cambio). Para la actividad económica se derivaron modelos de rezagos distribuidos simples. A partir de esos ejercicios se encontró que los agregados monetarios también ayudan a predecir de modo marginal.

El resto del documento se organizó como sigue. En la sección 2 se describen las series utilizadas y su grado de integración. La sección 3 presenta el marco conceptual. La sección 4 presenta los estimados de los parámetros de largo plazo y las pruebas de estabilidad. La sección 5 contiene la derivación y análisis de los mecanismos de corrección de error. La sección 6 presenta el 
análisis del contenido informativo de los agregados monetarios para explicar y predecir a la inflación y a la actividad económica. La sección 7 contiene las conclusiones y los comentarios finales.

\section{Descripción de las Series}

Las series utilizadas tienen una periodicidad mensual, comienzan en enero de 1980 y terminan en enero de 2001. Los datos de los agregados monetarios y de la tasa de interés de los CETES a 28 días provienen de los indicadores económicos del Banco de México. La variable de escala utilizada es el índice de la producción industrial generado por el INEGI. Las letras mayúsculas indican los valores corrientes de las series mientras que las letras minúsculas representan logaritmos. Todas las series utilizadas se consideran $I(1)$ (es decir, variables a las que se debe de sacar primeras diferencias para obtener series con media y varianza constante) aunque el Cuadro 1 de pruebas de raíces unitarias tiene resultados ambiguos, dependiendo de la prueba considerada. Tres asteriscos indican significancia al $1 \%$, dos de ellos al 5\% y un asterisco al $10 \%$.

\section{Cuadro 1.}

Pruebas de Raíces Unitarias

\begin{tabular}{|c|c|c|c|c|c|}
\hline \multirow[t]{2}{*}{ Variable } & \multicolumn{3}{|c|}{ Dickey-Fuller Aumentada } & \multicolumn{2}{|c|}{ Pillips-Perron } \\
\hline & Especif. & Rez. & Estad. & Especif. & Estad. \\
\hline \multicolumn{6}{|c|}{ Niveles } \\
\hline$\overline{m y b}$ & $\overline{\mathrm{C}}$ & 12 & -1.93 & $\overline{\mathrm{C}}$ & -2.39 \\
\hline $\mathrm{m} 1$ & T y C & 12 & -2.12 & $\mathrm{~T}$ y $\mathrm{C}$ & -1.95 \\
\hline $\mathrm{m} 2$ & T y C & 12 & -2.37 & $\mathrm{C}$ & 0.496 \\
\hline $\mathrm{m} 3$ & T y C & 12 & -1.93 & T y C & -1.69 \\
\hline $\mathrm{m} 4$ & $\mathrm{~T}$ y $\mathrm{C}$ & 12 & -1.93 & $\mathrm{~T}$ y C & -1.74 \\
\hline $\mathrm{p}$ & $\mathrm{C}$ & 11 & -2.52 & T y C & 0.18 \\
\hline y & T y C & 12 & -2.17 & T y C & $-3.58 * *$ \\
\hline $\mathrm{i}$ & $\mathrm{N}$ & 8 & -0.83 & $\mathrm{~N}$ & -1.31 \\
\hline \multicolumn{6}{|c|}{ Diferencias } \\
\hline myb & $\overline{\mathrm{N}}$ & 11 & -0.93 & $\overline{\mathrm{N}}$ & $-14.52 * * *$ \\
\hline $\mathrm{m} 1$ & $\mathrm{C}$ & 11 & -1.04 & $\mathrm{C}$ & $-15.69 * * *$ \\
\hline $\mathrm{m} 2$ & $\mathrm{~N}$ & 11 & -1.04 & $\mathrm{~N}$ & $-9.08 * * *$ \\
\hline $\mathrm{m} 3$ & $\mathrm{C}$ & 12 & -0.85 & $\mathrm{C}$ & $-15.56 * * *$ \\
\hline $\mathrm{m} 4$ & $\mathrm{C}$ & 12 & -0.97 & $\mathrm{C}$ & $-15.56 * * *$ \\
\hline $\mathrm{p}$ & $\mathrm{N}$ & 12 & -1.43 & $\mathrm{C}$ & $-3.82 * * *$ \\
\hline$y$ & $\mathrm{C}$ & 12 & $-3.45 * * *$ & $\mathrm{C}$ & $-29.35 * * *$ \\
\hline $\mathrm{i}$ & $\mathrm{N}$ & 7 & $-7.44 * * *$ & $\mathrm{~N}$ & $-10.42 * * *$ \\
\hline \multicolumn{6}{|c|}{ *** $1 \%$ de significancia } \\
\hline \multicolumn{6}{|c|}{$* * 5 \%$ de significancia } \\
\hline \multicolumn{6}{|c|}{$\mathrm{T}$ y $\mathrm{C}=$ Tendencia y Constante } \\
\hline \multicolumn{6}{|c|}{$\mathrm{C}=$ Constante } \\
\hline \multicolumn{6}{|c|}{$\mathrm{N}=\mathrm{Nada}$ (sin tendencia ni Constante) } \\
\hline
\end{tabular}

La prueba ADF clasifica a los agregados monetarios y al índice de precios como variables I(2); Sin embargo, la prueba de Phillips-Perron los clasifica como I(1). Al índice de precios se le 
puede clasificar también como I(1) si se deja una tendencia determinística en la especificación. Esta ambigüedad resulta de que la muestra utilizada ${ }^{4}$ es pequeña. El índice de la producción industrial $y$ presenta una situación similar ya que la primera de estas pruebas lo clasifica como $I(1)$ y la segunda como $I(0)$. Considerar a todas la series como $I(1)$ permitió obtener resultados más satisfactorios, aunque esto de ninguna manera puede considerarse como evidencia definitiva sobre el grado real de integración de aquellas variables. El uso del análisis $I(1)$ puede justificarse, además, por el hecho de que las ecuaciones de demanda de dinero se derivan para dinero real (tal como la teoría lo sugiere) y esta variable contiene una sola raíz unitaria para cada agregado.

\section{Marco Teórico}

Existe un amplio conjunto de enfoques teóricos que relacionan a la demanda de dinero con sus determinantes. Para la presente investigación se eligió una forma funcional sumamente simple, que trabaja muy bien. En la misma, la demanda de dinero de largo plazo se expresa en términos reales y depende de una variable de escala y de una medida del propio costo de oportunidad. ${ }^{5}$ Específicamente, la relación a estimar tiene la forma ${ }^{6}$ :

$$
\frac{M_{t}}{P_{t}}=\mu Y_{t}^{\gamma} e^{\varphi i_{t}}
$$

En este trabajo $\frac{M}{P}$ es la demanda real de algún agregado monetario, $Y$ la variable de escala (generalmente el índice de la producción industrial) e $i$ alguna tasa de interés. ${ }^{7}$ Los parámetros de largo plazo $\gamma$ y $\varphi$ son respectivamente la elasticidad ingreso y la semi-elasticidad de la tasa de interés. Por otra parte, $\mu$ es una constante. Para que la ecuación (1) sea interpretable

\footnotetext{
${ }^{4}$ Puede demostrarse con datos de 1940 a 2000 que el índice de precios al mayoreo de la Ciudad de México es una variable $I(1)$. Cabe aclarar que el índice de precios al consumidor comenzó a elaborarse apenas desde 1969.

${ }^{5}$ Algunas especificaciones teóricas incluyen el costo de oportunidad de mantener activos alternativos.

${ }^{6}$ Un intento reciente de formulación alternativa es el de Khamis y Leone (1999). Dichos autores quienes incluyen a la inflación como uno de los determinantes del costo de oportunidad a pesar de que sus pruebas muestran que esta última variable no es del mismo orden de integración que las demás. Ellos encuentran dos vectores de cointegración aunque la ecuación de corto plazo que desarrollan para la demanda de dinero no incluye entre las variables explicativas al segundo término de corrección de error.

${ }^{7}$ Estrictamente hablando, el costo de oportunidad es distinto para cada agregado. Para los billetes y monedas, el costo es la mejor tasa de interés que deja de percibirse, mientras que para un agregado más amplio tendría que restarse el rendimiento promedio de los instrumentos que conforman el agregado. Razones de disponibilidad de datos evitan poder usar medidas más precisas que la utilizada aquí (la tasa de los CETES de 28 días).
} 
como una demanda de dinero, la teoría económica sugiere las siguientes restricciones: $\gamma>0$ y $\varphi<0 .{ }^{8}$ A menudo la ecuación (1) es reescrita en logaritmos por razones prácticas:

$$
m_{t}-p_{t}=\ln (\mu)+\gamma y_{t}+\varphi i_{t}
$$

Para la discusión empírica se considera exclusivamente esta última forma.

\section{Estimación y Estabilidad de las Relaciones de Largo Plazo}

Por su importancia, la obtención de una demanda de dinero ha sido uno de los problemas empíricos más conocidos en economía. Este consiste en encontrar una fórmula sencilla de relacionar la cantidad de dinero deseada por el público con unas cuantas variables explicativas relevantes. La utilidad de dicha relación depende de cuán robusta sea ésta ante cambios en el entorno económico. Una función de demanda de dinero que cambie ante cada evento económico sería de escasa o nula utilidad. Por esta razón, la obtención de una demanda de dinero no concluye con la estimación de sus parámetros. Ello, toda vez que también es necesario comprobar su estabilidad, es decir, que los parámetros no se modifiquen durante el período de análisis.

Las primeras pruebas de estabilidad que se aplicaron en este trabajo fueron pruebas simples de Chow de cambio estructural. El siguiente paso fue la aplicación de baterías completas de pruebas a modelos de corrección de error, las cuales se realizan en la sección 5 de este trabajo. La práctica más reciente es la de probar, además, la estabilidad de la relación de largo plazo. Debido a que la estimación de los parámetros de dicha relación ${ }^{9}$ se lleva a cabo con métodos de cointegración, son necesarias algunas pruebas especiales las cuales se explican brevemente más adelante.

\footnotetext{
${ }^{8}$ Las restricciones toman a veces valores específicos como en el caso de la teoría cuantitativa con $\gamma=1$ o la ley de la raíz cuadrada donde $\gamma=0.5$ y $\varphi=0.5$.

${ }^{9}$ Es decir, de los parámetros $\mu, \gamma$ y $\varphi$ de la ecuación 2.
} 


\section{IV.1 Resultados}

La relación 2 para cada agregado se estimó con diferentes procedimientos. Todos ellos llevaron a resultados similares en cuanto a los valores de los parámetros estimados. En este documento se reportan principalmente los resultados del método de mínimos cuadrados ordinarios completamente modificados (MCO-CM) de Phillips y Hansen (1990), aunque a veces se hace referencia a los resultados obtenidos con el método de máxima verosimilitud de Johanssen (1988).

El primero permite la realización de pruebas de hipótesis basadas en el principio del cociente de verosimilitud. Por otra parte, el segundo es más robusto ante desviaciones del supuesto de normalidad en muestras pequeñas y, más importante, permite la aplicación de pruebas sencillas de estabilidad sobre la relación de largo plazo. Este último aspecto se encuentra prácticamente ausente de la literatura sobre la demanda de dinero. ${ }^{10}$

Se corrieron regresiones de la cantidad nominal de dinero contra precios, tasa de interés y actividad económica. Los resultados dieron coeficientes cercanos a la unidad para precios y valores razonables para las otras dos variables. Por esta razón se decidió llevar a cabo el análisis en términos de la cantidad real de dinero, enfoque que produce funciones de largo plazo estables de acuerdo a las pruebas utilizadas aquí.

Las estimaciones que se reportan a continuación en el Cuadro 2 provienen de la utilización del método MCO-CM de Hansen y Phillips (1989). El período de estimación para todos los agregados, salvo para billetes y monedas, fue de enero de 1980 a enero de 2001. Para billetes y monedas no fue posible obtener estimados razonables con este método sino a partir de $1983 .{ }^{11} \mathrm{La}$ estimación con MCO-CM está basada en el supuesto de que hay un único vector de cointegración en cada sistema. Las pruebas de Johansen (no reportadas aquí pero que pueden ser requeridas al autor) validan dicha hipótesis tanto para la cantidad nominal de dinero como para la cantidad real de dinero. En las columnas 2 a 4 se presentan los coeficientes de largo plazo estimados y debajo de cada uno de ellos, entre paréntesis, se muestran los errores estándar modificados según el método de MCO-CM.

\footnotetext{
${ }^{10}$ Las únicas excepciones conocidas son Gabriel et al.(1999) y Vega(2000).
} 
En las columnas 5 a 7 se incluyen tres estadísticos de constancia global de la relación de largo plazo. El primero y segundo estadísticos (LC y F promedio) tienen como hipótesis nula un vector de parámetros constantes contra la alternativa de un vector que cambia aleatoriamente en el tiempo. El tercer estadístico ( $\mathrm{F}$ supremo) tiene a la misma hipótesis nula contra la alternativa de un cambio único en el vector de cointegración en una fecha desconocida. Debajo del valor del estadístico, se encuentra el valor $p$ asintótico del mismo. ${ }^{12}$ A veces estas pruebas de estabilidad arrojan resultados que se contradicen entre sí. El criterio utilizado fue el de ponderar cada una de ellas de manera equitativa, es decir, si dos estadísticos rechazan la hipótesis nula de inestabilidad entonces se considera que la ecuación es estable.

\section{Cuadro 2.}

\section{Elasticidades de Largo Plazo y Pruebas de Estabilidad}

\begin{tabular}{|c||c|c|c||c|c|c|}
\hline \multirow{2}{*}{$\begin{array}{c}\text { Agregados } \\
\text { Monetarios }\end{array}$} & \multicolumn{3}{|c||}{ Elasticidades de Largo Plazo* } & \multicolumn{3}{c|}{ Pruebas de Estabilidad** } \\
\cline { 2 - 4 } & $\mathrm{i}$ & $\mathrm{y}$ & Constante & $\mathrm{LC}$ & $\mathrm{F}$ prom. & F.sup. \\
\hline \hline$m y b-p$ & -0.478 & 0.31 & 11.539 & 0.773 & 5.929 & 14.074 \\
& $(0.081)$ & $(0.139)$ & $(0.492)$ & $\mathrm{p}=0.024$ & $\mathrm{p}=0.060$ & $\mathrm{p}=0.074$ \\
\hline$m 1-p$ & -1.479 & 0.659 & 4.209 & 0.411 & 7.427 & 19.349 \\
& $(0.248)$ & $(0.415)$ & $(1.941)$ & $\mathrm{p}=0.161$ & $\mathrm{p}=0.023$ & $\mathrm{p}=0.010$ \\
\hline$m 2-p$ & -0.624 & 1.05 & 3.488 & 0.358 & 5.37 & 14.057 \\
& $(0.173)$ & $(0.295)$ & $(1.379)$ & $\mathrm{p}=0.200$ & $\mathrm{p}=0.089$ & $\mathrm{p}=0.077$ \\
\hline$m 3-p$ & -0.285 & 1.409 & 1.913 & 0.387 & 4.34 & 17.24 \\
& $(0.079)$ & $(0.134)$ & $(0.628)$ & $\mathrm{p}=0.184$ & $\mathrm{p}=0.174$ & $\mathrm{p}=0.022$ \\
\hline$m 4-p$ & -0.27 & 1.957 & -0.421 & 0.557 & 7.245 & 20.616 \\
& $(0.104)$ & $(0.177)$ & $(0.825)$ & $\mathrm{p}=0.071$ & $\mathrm{p}=0.026$ & $\mathrm{p}=0.010$ \\
\hline \hline
\end{tabular}

*Errores estándar en paréntesis

**p=valor $\mathrm{p}$ para el estadístico

Todas las estimaciones de los agregados comienzan en 1980 excepto myb-p que empieza en 1983

Para la estimación de la demanda de billetes y monedas en términos reales la ley de la raíz cuadrada de Tobin-Baumol parece estar muy cerca de cumplirse. ${ }^{13}$ La elasticidad ingreso fue de 0.31, inferior a la reportada por Khamis y Leone (1999) para el mismo agregado. ${ }^{14}$ Los valores $p$ de los estadísticos $\mathrm{F}$ promedio y $\mathrm{F}$ supremo no permiten rechazar la hipótesis de una relación de largo

\footnotetext{
${ }^{11}$ Los valores obtenidos con el método de Johanssen desde 1982 fueron una semielasticidad interés de -0.48 , elasticidad ingreso de 0.52 y constante de 10.41 . Evidentemente, estos valores son muy aproximados a los que se reportan en el texto.

${ }^{12}$ Un valor p proporciona el nivel mínimo de significancia al cual es posible rechazar la hipótesis nula. Por ejemplo, un valor $p$ de 0.07 indica que un estadístico es significativo al $10 \%$ pero no al $5 \%$.

${ }^{13}$ De hecho, estimaciones realizadas con el método de Johansen no rechazan que las elasticidades cumplan dicha ecuación.
} 
plazo constante para billetes y monedas desde 1983 a 2000 mientras que el estadístico LC indica rechazo al 5\%. En vista de los resultados anteriores, se tomó la decisión de que la relación de largo plazo para este agregado es estable desde 1983.

La elasticidad ingreso crece con el nivel del agregado. En el caso de M1, resultó de 0.66 mientras que la semielasticidad con respecto a la tasa de interés fue de -1.48. Este último parámetro es mayor que el estimador para cualquier otro agregado y refleja el efecto sobre la estimación del cambio en la definición de M1 a partir de octubre de $1991 .{ }^{15}$ A consecuencia de este cambio, solamente el estadístico LC no rechaza la hipótesis nula de estabilidad.

Para $m 2-p$ la elasticidad ingreso resultó unitaria y la semielasticidad de la tasa de interés es mucho menor que la del caso anterior. Así, ninguna de las pruebas permite rechazar la hipótesis nula de estabilidad del vector al 5\% de significancia.

El patrón se mantiene en el caso de m3-p para el cual la elasticidad ingreso aumenta y la semielasticidad de la tasa de interés disminuye. Para este agregado dos de las pruebas no rechazan la hipótesis de estabilidad. La ecuación para $m 4-p$ muestra una semielasticidad de tasa de interés muy similar a la de M3 aunque la elasticidad ingreso es bastante más alta. Este fue el único agregado para el cual dos de las pruebas reportan inestabilidad al $5 \%$.

De la discusión anterior se concluye que solamente es posible aceptar sin reservas la hipótesis de estabilidad de la relación de largo plazo de 1980 a 1999 para el caso de $m 2-p$. En cuanto a $m 3-p$ la evidencia de estabilidad para el mismo período fue menos fuerte pero razonablemente buena. Para billetes y monedas, el más básico de los agregados aquí considerados, la estabilidad de la demanda de dinero de largo plazo parece segura a partir de 1983 mientras que para $m 1-p$ y $m 4-p$ hay indicios de inestabilidad para segmentos bien definidos de la muestra. En cuanto a $m 1-p$ el problema parece provenir de una innovación financiera ${ }^{16}$ (cheques

\footnotetext{
${ }^{14}$ Obtenida mediante el método de Johansen, al igual que los valores reportados en el pie de página 10.

15“A partir de septiembre de 1991 tuvo lugar una transferencia masiva de fondos de los fideicomisos abiertos de inversión, incluyendo los de las cuentas maestras, hacia las cuentas de cheques con intereses. Lo anterior fue propiciado de manera determinante por modificaciones introducidas en las regulaciones relativas al coeficiente de liquidez y al régimen de inversión de los fideicomisos citados. De hecho, ya para diciembre las cuentas maestras habían desaparecido por completo. Este fenómeno explica, por sí mismo, un incremento del orden de 26.5 billones de pesos en las cuentas de cheques y en M1 en los últimos meses del año." Ver Banco de México (1991) Informe Anual.

${ }^{16}$ Es interesante recordar que fenómenos análogos han ocurrido en otros países. Por ejemplo, Hendry y Ericsson (1991, p.839) mencionan la "explosión de M1" a partir de mediados de los años ochenta como uno de los fenómenos a explicar en los Estados Unidos y en la Gran Bretaña. Así, dichos autores señalan la
} 
que pagan intereses) introducida en 1991 mientras que para $m 4$ - $p$ el problema parece ocurrir a partir de 1995, coincidiendo con el inicio de la crisis económica y la modificación del régimen cambiario.

\section{El Proceso de Ajuste}

Cada sistema cointegrador tiene asociada una representación del mecanismo de corrección de error, ${ }^{17}$ que en este caso puede interpretarse como la demanda de dinero de corto plazo para cada agregado. ${ }^{18}$ Dicho mecanismo representa el proceso de ajuste hacia el equilibrio de largo plazo que la demanda de saldos reales sigue ante perturbaciones en las variables explicatorias. El aspecto distintivo de un modelo de corrección de error es que incluye como variable explicativa a las desviaciones del equilibrio de largo plazo y también incorpora rezagos de las variables involucradas y factores estacionales, por lo que resulta un buen instrumento para hacer simulaciones de política y pronóstico. En esta sección se estiman y se someten a pruebas estadísticas los modelos de corrección de error para cada agregado.

\section{V.1 Especificación del Modelo de Corrección de Error}

Hendry y Ericsson (1991) señalan diversas restricciones para que el mecanismo de corrección de error sea interpretable como una demanda de dinero. En el modelo de ajuste, dado por:

$$
\Delta(m-p)_{t}=\mu_{0} C E_{t-1}+\mu_{1}(L) \Delta(m-p)_{t-1}+\mu_{2}(L) \Delta i_{t}+\mu_{3}(L) \Delta y_{t}
$$

se debe cumplir con lo siguiente: 1) $\mu<0$ (para que exista cointegración); 2) $\mu_{2}(1) \leq 0$ (la suma de los efectos de corto plazo de la tasa de interés debe ser negativa) y; $\mu_{3}(1) \geq 0$ (la suma de los efectos de corto plazo de la variable de escala debe ser positiva).

\section{V.2 Interpretación de los Estimados}

En el Cuadro 3 se muestran los coeficientes estimados de los modelos de corrección de error para cada una de las definiciones de dinero utilizadas en este documento (con la excepción de $m 2-p$, que se presenta en el Cuadro 4. Por brevedad, no se recogen los estimados de los coeficientes de variables estacionales. Los errores estándar reportados entre paréntesis son

necesidad de tomar en cuenta los procesos de innovación financiera para la obtención de funciones de demanda de dinero estables.

${ }^{17}$ Este es precisamente el Teorema de Representación de Granger (Engle y Granger 1988). 
congruentes según el método de White. Las columnas tres, cinco, siete y nueve contienen los estadísticos de Hansen (1991a) para probar la estabilidad individual de los parámetros de una regresión con variables estacionarias. ${ }^{19}$ Debajo de cada conjunto de estimados se presentan varios estadísticos útiles para la evaluación del ajuste y la especificación.

\section{Cuadro 3.}

Ecuaciones de Corto Plazo para Distintos Agregados Monetarios

\begin{tabular}{|c|c|c|c|c|c|c|c|c|}
\hline \multirow[t]{2}{*}{ Variables } & \multicolumn{2}{|c|}{$\Delta(\text { myb-p })_{t}$} & \multicolumn{2}{|c|}{$\Delta(\mathrm{m} 1-\mathrm{p})_{\mathrm{t}}$} & \multicolumn{2}{|c|}{$\Delta(\mathrm{m} 3-\mathrm{p})_{\mathrm{t}}$} & \multicolumn{2}{|c|}{$\Delta(\mathrm{m} 4-\mathrm{p})_{\mathrm{t}}$} \\
\hline & Coef. & Inest. & Coef. & Inest. & Coef. & Inest. & Coef. & Inest. \\
\hline $\mathrm{Ce}_{\mathrm{t}-1}$ & $\begin{array}{l}-0.067 \\
(0.015) \\
\end{array}$ & 0.08 & $\begin{array}{c}-0.041 \\
(0.008)\end{array}$ & 0.07 & $\begin{array}{c}-0.038 \\
(0.010)\end{array}$ & 0.06 & $\begin{array}{c}-0.038 \\
(0.010) \\
\end{array}$ & $0.71 *$ \\
\hline$\Delta(\mathrm{m}-\mathrm{p})_{\mathrm{t}-1}$ & & & $\begin{array}{c}0.151 \\
(0.064)\end{array}$ & 0.21 & $\begin{array}{c}0.169 \\
(0.063)\end{array}$ & 0.08 & $\begin{array}{c}0.261 \\
(0.060)\end{array}$ & 0.22 \\
\hline$\Delta(m-p)_{t-3}$ & $\begin{array}{c}0.140 \\
(0.063)\end{array}$ & 0.04 & & & & & & \\
\hline$\Delta \mathrm{i}_{\mathrm{t}}$ & $\begin{array}{l}-0.076 \\
(0.026) \\
\end{array}$ & 0.02 & $\begin{array}{l}-0.117 \\
(0.033)\end{array}$ & 0.08 & $\begin{array}{c}-0.073 \\
(0.018) \\
\end{array}$ & 0.25 & $\begin{array}{l}-0.067 \\
(0.017) \\
\end{array}$ & 0.05 \\
\hline$\Delta \mathrm{i}_{\mathrm{t}-1}$ & $\begin{array}{c}-0.068 \\
(0.029)\end{array}$ & 0.05 & & & & & & \\
\hline$\Delta \mathrm{y}_{\mathrm{t}}$ & $\begin{array}{l}-0.234 \\
(0.065) \\
\end{array}$ & 0.04 & & & $\begin{array}{c}0.183 \\
(0.052) \\
\end{array}$ & $\overline{0.04}$ & $\begin{array}{c}0.158 \\
(0.042) \\
\end{array}$ & 0.01 \\
\hline$\Delta \mathrm{y}_{\mathrm{t}-1}$ & & & & & $\begin{array}{c}0.144 \\
(0.062)\end{array}$ & 0.16 & & \\
\hline$\Delta \mathrm{y}_{\mathrm{t}-2}$ & & & & & $\begin{array}{c}0.165 \\
(0.061)\end{array}$ & 0.09 & & \\
\hline$\Delta \mathrm{y}_{\mathrm{t}-3}$ & $\begin{array}{c}0.142 \\
(0.066) \\
\end{array}$ & 0.13 & & & $\begin{array}{c}0.104 \\
(0.053) \\
\end{array}$ & $\overline{0.04}$ & & \\
\hline$\overline{\mathrm{R}^{2}}$ & $\overline{0.8}$ & & & & & & & \\
\hline R-ajust & 0.8 & & & & & & & \\
\hline E.E. & 0.0 & & & & & & & \\
\hline Asimetría & -0. & & & & 1.0 & & & \\
\hline Curtosis & 4.9 & & & & & & & \\
\hline Jarque-Bera & 43.0 & & 3948 & $27 * *$ & 586.0 & $19^{* *}$ & 604. & $1 * *$ \\
\hline $\mathrm{LM}_{12} \mathrm{AR}$ & 0.6 & & & & 0.8 & & & \\
\hline $\mathrm{LM}_{12} \mathrm{ARCH}$ & 1.3 & & & & 0.4 & & & \\
\hline White-Het. & 3.23 & & & & & & & \\
\hline Reset & 1.1 & & & & 6.76 & & 12. & $7 * *$ \\
\hline
\end{tabular}

*** $1 \%$ de significancia

** $5 \%$ de significancia

* $10 \%$ de significancia

\footnotetext{
${ }^{18}$ Como en Hendry y Ericsson (1991).
}

${ }^{19}$ Estas pruebas de estabilidad no deben confundirse con las ecuaciones reportadas en el Cuadro 2, las cuales prueban la estabilidad conjunta de los parámetros de una regresión con variables $I(1)$. 
El enfoque seguido para la obtención de estos modelos fue el llamado "general a específico," por lo cual todos los coeficientes incluidos tienen garantizada su presencia por su nivel de significancia o alguna otra razón estadística. Los asteriscos en las pruebas de especificación implican el rechazo de la hipótesis nula que se está probando (por ejemplo para el estadístico Jarque-Bera la hipótesis nula es que la distribución es normal). Tres asteriscos indican rechazo al $1 \%$, dos al $5 \%$ y uno al $10 \%$. Para simplificar la notación, los términos de corrección de error y rezagos de las variables dependientes se designan genéricamente como $C E_{t-1}$ y $\Delta(m-p)_{t-i}$, respectivamente. En todas las ecuaciones el coeficiente para el término de corrección de error rezagado $C E_{t-1}$ salió con el signo correcto y resultó altamente significativo, comprobando la propiedad de cointegración. Estos coeficientes se vuelven menos negativos al aumentar la agregación. En el Cuadro 4 se muestra cuántos meses son requeridos para que una perturbación al equilibrio monetario de cada agregado se elimine en un 50\% y un $95 \%$. La velocidad de ajuste para $\Delta(m 4-p)_{t-i}$ es la misma que para $\Delta(m 3-p)_{t-i}$.

\section{Cuadro 4.}

\section{Velocidades de Ajuste}

\begin{tabular}{|l||c|c|c|}
\hline Variables & CE & $50 \%$ & $95 \%$ \\
\hline \hline$\Delta($ myb-p $)$ & -0.067 & 11 & 46 \\
$\Delta(\mathrm{m} 1-\mathrm{p})$ & -0.041 & 17 & 75 \\
$\Delta(\mathrm{m} 2-\mathrm{p})$ & -0.043 & 17 & 71 \\
$\Delta(\mathrm{m} 3-\mathrm{p})$ & -0.038 & 19 & 80 \\
\hline \multicolumn{2}{|l|}{ Las columnas 3 y 4 representan meses }
\end{tabular}

Como puede observarse, el equilibrio en el agregado más básico se restablece en cerca de la mitad del tiempo que en el agregado más amplio. Para $m y b-p$ toma alrededor de cuatro años eliminar 95\% de una perturbación, mientras que para $m 3-p$ toma 80 meses (6.6 años). De lo anterior se concluye que el agregado más básico posee una velocidad de ajuste considerablemente más alta que la de los agregados amplios. Sin embargo, esta velocidad de ajuste es lenta si el agregado más básico quisiera utilizarse como objetivo intermedio de política monetaria.

Los coeficientes de los términos autorregresivos, $\Delta(m-p)_{t-i}$, son bajos para todos los casos. Los efectos de corto plazo de la tasa de interés tienen el signo negativo necesario para que las funciones sean interpretadas como ecuaciones de demanda de dinero. El cambio contemporáneo de 
la tasa de interés está presente en cada caso y sólo en la versión de $m y b-p$ aparece también con un rezago. Esto implica que la demanda real de dinero comienza a reaccionar de inmediato ante una perturbación en la tasa de interés.

Los cambios contemporáneos en la variable de escala, $\Delta y_{t}$, solamente resultaron no significativos para el caso de $\Delta(m 1-p)_{t-i}$. Algunos rezagos de esta variable aparecen en algunas de las ecuaciones. El signo negativo de la suma de los coeficientes autorregresivos en la primera ecuación es anómalo en términos de las restricciones señaladas por Hendry y Ericsson (1991).

\section{V.3 Pruebas de Especificación y Bondad de Ajuste}

La ecuación con el mejor ajuste (medido por la $\bar{R}^{2}$ ) resultó la correspondiente al agregado básico (0.867) y el ajuste se hace menos robusto al aumentar el grado de agregación. Congruente con lo anterior, la ecuación con el menor error estándar es la de $\Delta(m y b-p)_{t-i}$, lo que augura menor incertidumbre al ser usada para preparar pronósticos.

Obsérvese que en ninguna de las regresiones se incluyeron variables dicótomicas aunque parece tentador hacerlo para eliminar el problema de no normalidad de los residuales que aparece en todas las ecuaciones. Por ejemplo, la distribución de errores más asimétrica y leptocúrtica es la que proviene de la regresión para $\Delta(m 1-p)_{t-i}$. La razón de esto es una observación extrema en septiembre de 1991 (ya mencionada).

La introducción de una variable dicotómica para manejar estadísticamente dicho evento permitió aumentar el ajuste y reducir la no normalidad de modo significativo. El mismo procedimiento puede seguirse para eliminar el efecto de otros eventos extremos que producen la no normalidad. Una medida alternativa sería obtener estimados con una periodicidad trimestral, por medio de lo cual se eliminaría el problema de no normalidad en muchos de los $\operatorname{casos}^{20} \mathrm{La}$ inferencia estadística con dichas variables dicotómicas es muy similar a la presentada aquí, por lo cual se omite su explicación por brevedad.

Ninguna de las ecuaciones presentó problemas de autocorrelación y efectos $\mathrm{ARCH}$, aunque para los dos primeros agregados mediante la prueba de White se rechazó la hipótesis de varianza constante. La prueba de especificación RESET fue superada por los dos primeros agregados pero no por los últimos dos.

\footnotetext{
${ }^{20}$ Por ejemplo, en Garcés(2001).
} 


\section{V.4 Pruebas de Constancia de los Parámetros Individuales y de las Ecuaciones}

En esta sección se discuten las pruebas de estabilidad y constancia para los procesos de ajuste. Estas complementan el análisis de estabilidad de las relaciones de largo plazo llevado a cabo en la sección 4. Las pruebas de esta sección son más conocidas y numerosas que las de la sección 4 pues son las que comúnmente se aplican a regresiones de MCO con series estacionarias.

La estabilidad individual de todos los coeficientes estimados se verifica mediante los estadísticos de las columnas tres, cinco, siete y nueve del Cuadro 3. La única excepción fue la del coeficiente de la velocidad de ajuste para el último agregado. Esto probablemente refleja los problemas de estabilidad en la relación de largo plazo. Un punto interesante es que ningún coeficiente para la ecuación de $\Delta(m 1-p)$ mostró signos de inestabilidad a pesar de los resultados para la relación de largo plazo. Este caso ejemplifica la necesidad de confirmar separadamente la estabilidad de los coeficientes de corto y largo plazos pues los primeros pueden resultar estables (en términos de alguna prueba) aún cuando los segundos no lo sean. En la Gráfica 1 se muestran los estimados recursivos de los coeficientes para la demanda de $\Delta(m y b-p)$.

\section{Gráfica 1.}

\section{Coeficientes Recursivos para $\Delta(m y b-p)$.}
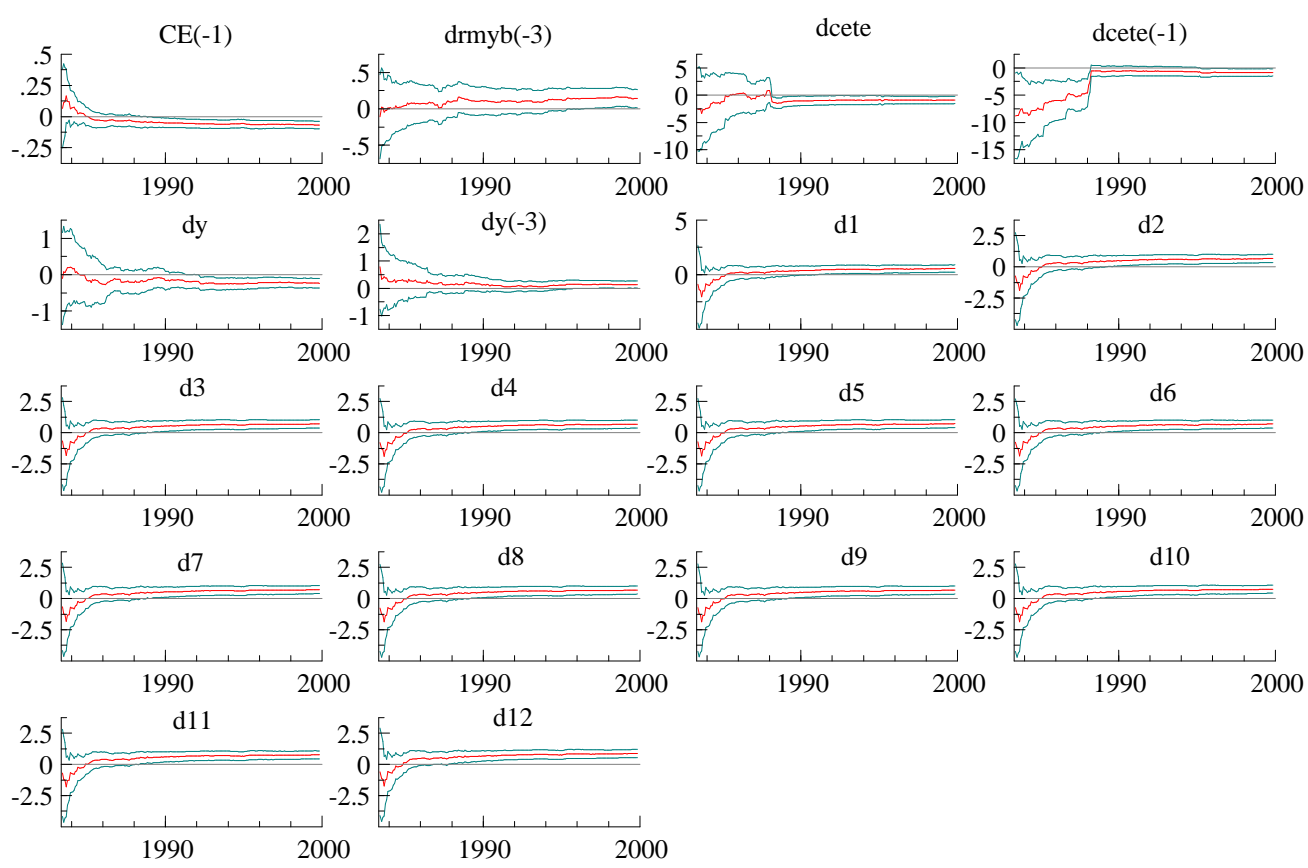
La apariencia de las secuencias de estimados recursivos exhibe una extraordinaria constancia para la mayor parte de los coeficientes. Las únicas situaciones para las cuales las sendas temporales no resultaron suaves ocurren al comienzo de 1988, cuando los coeficientes de corto plazo de la tasa de interés presentan un pequeño salto. Sin embargo, estos movimientos bruscos no son suficientes para rechazar la hipótesis de constancia de los parámetros, como se muestra en la tercera columna del Cuadro 5. En pocos de los casos restantes la estabilidad de los coeficientes fue tan buena como en la ecuación de $\Delta(m y b-p)$.

En la Gráfica 2 se muestran las secuencias temporales de algunos estadísticos recursivos para juzgar la estabilidad global de la ecuación para $\Delta(m y b-p)$. En el primer panel se presenta la suma de los cuadrados de los residuales (SCR); en el segundo, los residuales de un paso adelante con bandas de confianza de dos errores estándar; y en los últimos tres los estadísticos de Chow de un paso adelante.

\section{Gráfica 2.}

\section{Estadísticos Recursivos de Evaluación Global para $\Delta(m y b-p)$}
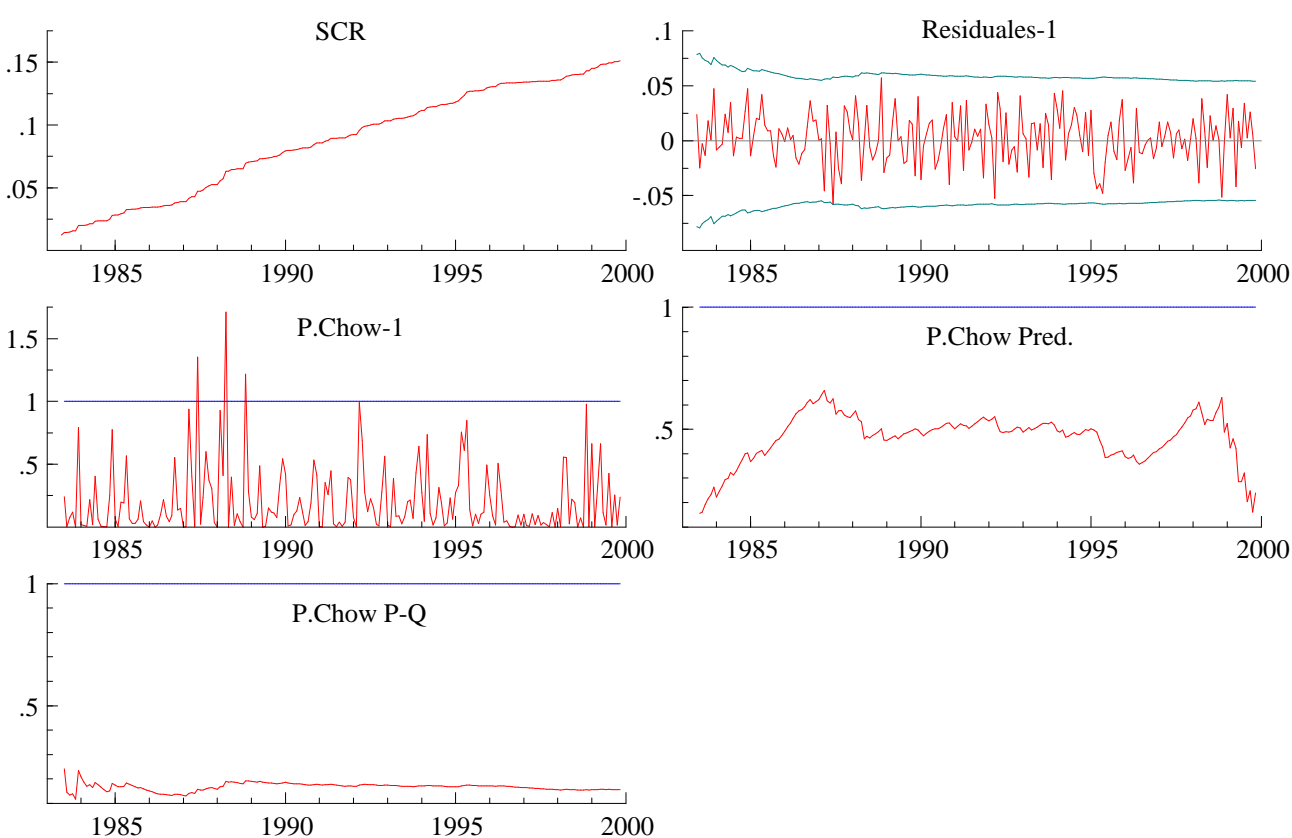
Los resultados confirman claramente la estabilidad global del modelo: la suma recursiva de los residuales al cuadrado es casi una línea recta; los residuales recursivos no salen de las bandas y solamente los estadísticos de Chow de un paso adelante (en la gráfica denotados como P.Chow-1) muestran observaciones que se apartan en tres ocasiones de la línea de significancia del 5\% (normalizada a 1). El análisis gráfico del resto de las ecuaciones del Cuadro 2 (que puede pedirse al autor) muestra que las propiedades de estabilidad de corto plazo no son tan satisfactorias para algunos otros agregados.

\section{V.5 Exogeneidad e Invertibilidad}

Un problema empírico que se presenta a menudo es decidir si la existencia de demandas de dinero estables garantiza el uso de éstas para la obtención de, por ejemplo, ecuaciones de comportamiento o pronóstico para la inflación, la tasa de interés o la actividad económica. En otras palabras, el investigador debe decidir si, dada una ecuación de demanda de dinero estable, es posible una inversión de ella para representar el proceso que genera alguna de las variables que la determinan. Hendry y Ericsson (1991) arguyen que ésta es una práctica común a la que se recurre en macroeconomía pero que no está justificada en general.

Específicamente, suponiendo que se ha estimado una demanda de dinero como cualquiera del Cuadro 2, el analista puede derivar la impresión errónea de que de esas ecuaciones se puede despejar, por ejemplo, a la inflación o la tasa de interés y utilizar esas expresiones para hacer pronósticos o algún ejercicio similar. El error se origina cuando se olvida el hecho de que una regresión es la estimación de una función de variables aleatorias ${ }^{21}$ y no una expresión algebraica donde las variables pueden moverse libremente a ambos lados de la igualdad. ${ }^{22}$

Si una función es invertible para alguna variable, el traslado de ésta a cualquier lado de la igualdad no afecta las propiedades estadísticas de aquélla. Esto sugiere que, para probar si la invertibilidad es posible en el caso de la demanda de dinero, debe procederse como sigue: obtener regresiones para el dinero nominal, la inflación y la tasa de interés contra el resto de las variables que están incluidas en la función original y analizar las propiedades estadísticas de los modelos resultantes. Los casos para los cuales la inversión no es justificable deben mostrar problemas en las pruebas estadísticas comunes, tal y como se muestra a continuación.

\footnotetext{
${ }^{21}$ Por ejemplo, una media condicional

${ }^{22}$ Para una prueba sencilla de que en general lo anterior no es posible, ver Hendry y Ericsson (1991).
} 
En el Cuadro 5 se presentan los coeficientes estimados para tres modelos de corrección de error (con exclusión de las variables dicótomicas estacionales) estimados sobre el mismo conjunto de información. En el primero la variable dependiente ${ }^{23}$ es $\Delta(m 2-p)$. El segundo está formado por una de las ecuaciones "invertidas" donde la variable dependiente es $\Delta p_{t}$, a la cual se le añadió $\Delta m 2_{t}$ como variable explicativa. ${ }^{24}$ La tercera ecuación (también proveniente de una inversión) tiene a $\Delta i_{t}$ como variable dependiente.

\section{Cuadro 5.}

Modelos de Corto Plazo para $\Delta(m 2-p), \Delta p_{t}$ y $\Delta i_{t}$

\begin{tabular}{|c|c|c|c|c|c|c|}
\hline \multirow[t]{2}{*}{ Variables } & \multicolumn{2}{|c|}{$\Delta(\mathrm{m} 2-\mathrm{p})_{\mathrm{t}}$} & \multicolumn{2}{|c|}{$\Delta \mathrm{p}_{\mathrm{t}}$} & \multicolumn{2}{|c|}{$\Delta \mathrm{i}_{\mathrm{t}}$} \\
\hline & Coef. & Inest. & Coef. & Inest. & Coef. & Inest. \\
\hline $\mathrm{Ce}_{\mathrm{t}-1}$ & $\begin{array}{l}-0.043 \\
(0.009)\end{array}$ & 0.09 & $\begin{array}{c}0.073 \\
(0.006)\end{array}$ & $4.20 * *$ & $\begin{array}{c}0.007 \\
(0.002)\end{array}$ & 0.10 \\
\hline$\Delta(\mathrm{m} 2-\mathrm{p})_{\mathrm{t}-6}$ & $\begin{array}{c}0.173 \\
(0.063)\end{array}$ & 0.03 & $\begin{array}{c}-0.062 \\
(0.040)\end{array}$ & 0.04 & $\begin{array}{c}0.005 \\
(0.016)\end{array}$ & 0.28 \\
\hline$\Delta \mathrm{i}_{\mathrm{t}}$ & $\begin{array}{c}-0.65 \\
(0.274)\end{array}$ & 0.26 & $\begin{array}{c}0.61 \\
(0.173)\end{array}$ & 0.05 & & \\
\hline$\Delta \mathrm{m} 2_{\mathrm{t}}$ & & & $\begin{array}{c}0.286 \\
(0.038)\end{array}$ & $.076 * *$ & & \\
\hline$\Delta(\mathrm{m} 2-\mathrm{p})_{\mathrm{t}}$ & & & & & $\begin{array}{l}-0.028 \\
(0.016)\end{array}$ & 0.09 \\
\hline$\overline{R \text {-ajust }}$ & \multicolumn{2}{|c|}{0.433} & \multicolumn{2}{|c|}{0.615} & \multicolumn{2}{|c|}{0.094} \\
\hline $\mathrm{R}^{2}$ & \multicolumn{2}{|c|}{0.468} & \multicolumn{2}{|c|}{0.64} & \multicolumn{2}{|c|}{0.036} \\
\hline E.E. & \multicolumn{2}{|c|}{0.024} & \multicolumn{2}{|c|}{0.153} & \multicolumn{2}{|c|}{0.006} \\
\hline Asimetría & \multicolumn{2}{|c|}{0.425} & \multicolumn{2}{|c|}{0.827} & \multicolumn{2}{|c|}{-4.492} \\
\hline Curtosis & \multicolumn{2}{|c|}{9.156} & \multicolumn{2}{|c|}{9.807} & \multicolumn{2}{|c|}{52.421} \\
\hline Jarque-Bera & \multicolumn{2}{|c|}{$373.330 * *$} & \multicolumn{2}{|c|}{$57.980 * *$} & \multicolumn{2}{|c|}{$24390 * *$} \\
\hline $\mathrm{LM}_{12} \mathrm{AR}$ & \multicolumn{2}{|c|}{0.933} & \multicolumn{2}{|c|}{$2.630^{* *}$} & \multicolumn{2}{|c|}{$3.744 * *$} \\
\hline $\mathrm{LM}_{12} \mathrm{ARCH}$ & \multicolumn{2}{|c|}{1.076} & \multicolumn{2}{|c|}{$30.020 * *$} & \multicolumn{2}{|c|}{0.302} \\
\hline White-Het. & \multirow{2}{*}{\multicolumn{2}{|c|}{1.605}} & \multicolumn{2}{|c|}{$6.090 * *$} & \multicolumn{2}{|c|}{0.869} \\
\hline Reset & & 0.419 & \multicolumn{2}{|c|}{$72.480 * *$} & \multicolumn{2}{|c|}{1.751} \\
\hline
\end{tabular}

*** $1 \%$ de significancia

** $5 \%$ de significancia

* $10 \%$ de significancia

\footnotetext{
${ }^{23}$ Se eligió $\Delta(m 2-p)$ por ser la ecuación más parsimoniosa, aunque ejercicios similares a éste para los otros agregados fueron llevados a cabo con resultados similares.

${ }^{24}$ Una ecuación con $\Delta m 2$ como variable dependiente tiene propiedades similares a la establecida en términos reales.
} 
El primer resultado que cabe destacar es que, salvo por el problema de no normalidad detectado mediante el estadístico Jarque-Bera, la ecuación para $\Delta m 2$ pasa todas las pruebas de especificación. Por otra parte, las ecuaciones "invertidas" presentan más problemas (en especial la correspondiente a la inflación, no pasa una sóla de las pruebas) y presentan un error estándar mucho mayor que el de aquélla. También es de notarse que todos los coeficientes de la ecuación de $\Delta(m 2-p)$ son estables, mientras que en la ecuación de $\Delta p$ para dos de ellos se rechaza fuertemente la hipótesis de estabilidad.

En la Gráfica 3 se presentan las secuencias de estadísticos recursivos de Chow de punto de quiebre para las tres ecuaciones anteriores. En ellas se muestra que la única ecuación estable es la que incorpora a $\Delta(m 2-p)$ como variable dependiente (en los dos paneles superiores). Las secuencias de los estadísticos para las otras dos ecuaciones cruzan la línea de significancia.

\section{Gráfica 3.}

Estadísticos Recursivos de Chow para $\Delta(m 2-p), \Delta p_{t} \mathbf{y} \Delta i_{t}$
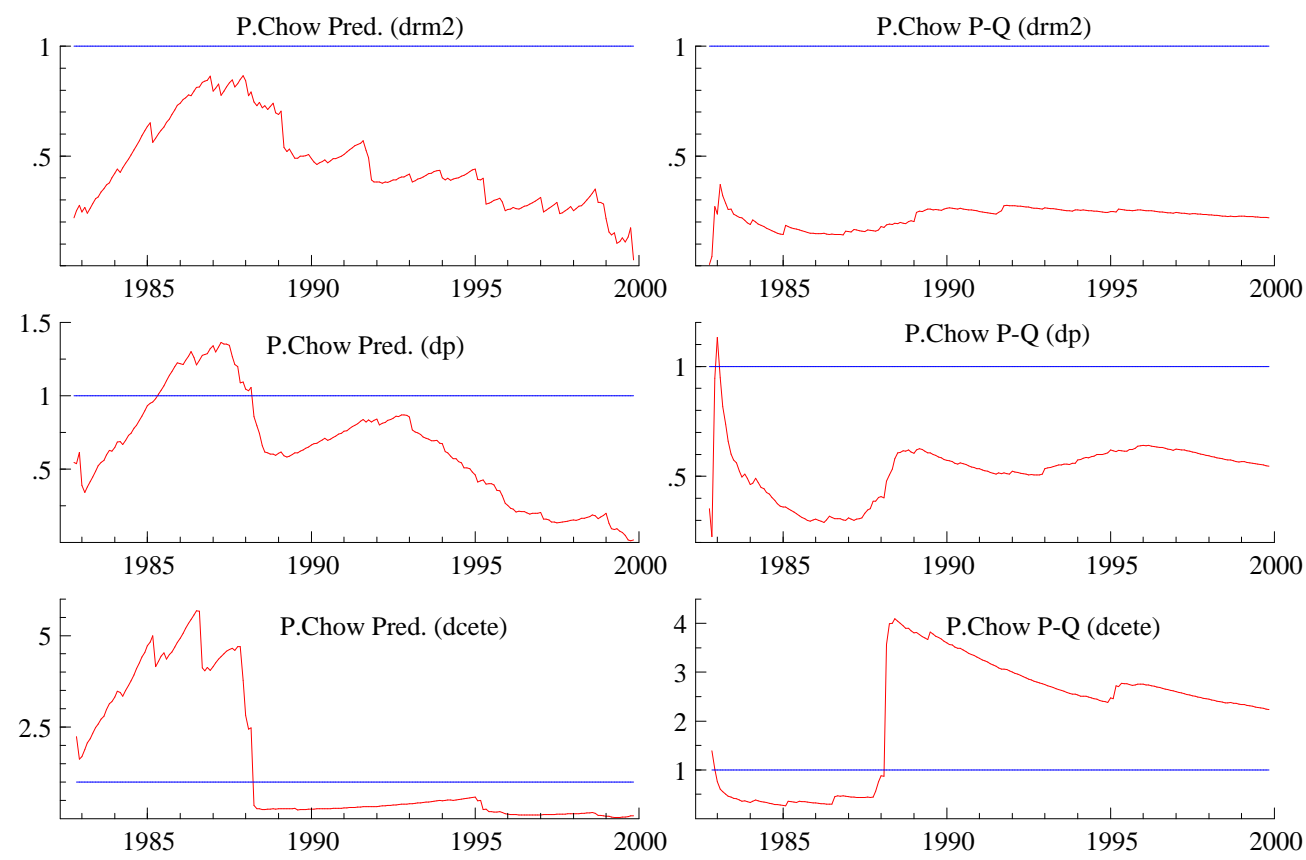

Los resultados anteriores sugieren que la ecuación de demanda de dinero no es invertible. Por esta razón no resulta posible obtener una ecuación de determinación de la inflación o de la tasa de interés a partir de una demanda de dinero. De este resultado se infiere que no hay implicaciones 
de política que se deriven directamente de una demanda de dinero estable. Aquéllas requieren, además, modelos bien especificados de la inflación, la tasa de interés y el producto. ${ }^{25}$

La no invertibilidad es una consecuencia de la superexogeneidad de una variable. ${ }^{26}$ Otra implicación de esta propiedad es que los parámetros estimados permanecen inalterados aún en presencia de cambios en el proceso seguido por las variables sobre las que se condiciona. Respecto a ello, se estimaron modelos uniecuacionales para los cambios de la tasa de interés y de la inflación (en poder del autor) que muestran que los procesos seguidos por dichas variables no son constantes. El de la tasa de interés parece constante después de marzo de $1988^{27}$ mientras que el de la inflación lo es al menos a partir de 1983 .

Es muy probable que tanto la inflación como la tasa de interés sean variables superexógenas en la ecuación de demanda de dinero. Para la tasa de interés, el asunto es más claro debido a que el proceso que sigue no es constante durante la muestra. ${ }^{28}$ En cuanto a la inflación, la no constancia del proceso marginal no es tan concluyente.

Es necesario aclarar que aún cumpliéndose la propiedad de superexogeneidad de una variable, ello no implica que la variable endógena no pueda utilizarse para hacer, por ejemplo, pronósticos sobre la otra variable. ${ }^{29}$ Este caso se ejemplifica en la sección 6

\footnotetext{
${ }^{25}$ Hendry y Ericsson (1991).

${ }^{26}$ Hendry y Ericsson (1991). Esta propiedad requiere de tres requisitos: exogeneidad débil (es decir, la validez del procedimiento de condicionar en cierta variable); constancia del modelo; e invariancia de los parámetros.

27 "Desde el otoño de 1988, las autoridades monetarias trataron de seguir un enfoque pragmático con respecto a las tasas de interés, dejando que el mercado funcionara. Con respecto a la eliminación gradual de los controles cuantitativos de crédito, la segunda fase del proceso de liberalización financiera se inició en 1988, con la eliminación de los 'cajones de crédito' a sectores de alta prioridad, y continuando con la supresión de los requisitos de reserva obligatoria. De esta manera, a partir de octubre de 1988 sólo los saldos bancarios de las cuentas de cheques y de ahorro estuvieron sujetos a limitaciones relacionadas con el porcentaje que debería canalizarse a 'sectores de alta prioridad' y a los bancos de desarrollo, además del requisito de reserva obligatoria [...]. En abril de 1989 se extendió la reforma a los depósitos a plazo, y en agosto sucedió lo mismo con las cuentas de cheques. Para agosto, las autoridades abandonaron el mecanismo de crédito selectivo y establecieron las condiciones para eliminar lo que quedaba del sistema de reserva mínima [...]. Con la implantación de estas medidas, el proceso de liberalización de un sistema financiero reprimido para lograr un esquema basado en principios de mercado y de transparencia quedó esencialmente concluido" (Pedro Aspe, El camino de la transformación económica de México, México, FCE, 1993).

${ }^{28}$ Por el cambio institucional de 1988 descrito anteriormente.

${ }^{29}$ En otras palabras, superexogeneidad no implica exogeneidad fuerte.
} 


\section{El Contenido Informativo de los Agregados Monetarios para el Pronóstico de la Inflación y la Actividad Económica}

En la sección 5.5 se ha mostrado que para el caso de México es imposible partir de una demanda de dinero y obtener ecuaciones de inflación, producto y tasa de interés. Esto no es lo mismo que afirmar que la información contenida en los agregados monetarios no puede utilizarse de algún modo para ayudar a la preparación del pronóstico para esas variables. En esta sección se explora dicha información en contextos diferentes para cada una de las variables consideradas.

\section{VI.1 Agregados Monetarios e Inflación}

Para la inflación se estimaron modelos basados en una versión simple del modelo $P^{*}$ de Hallan, Porter y Small (1991). En este modelo se define un nivel de precios de equilibrio a partir de la ecuación cuantitativa del dinero. La especificación es la que sigue:

$$
P_{t}^{*}=\frac{M_{t} V_{t}^{*}}{Y_{t}^{*}}
$$

en donde los asteriscos indican los valores de equilibrio del nivel de precios, de la velocidad y del producto. Nótese que en esta formulación, implícitamente se interpreta a $M_{t}$ como una variable de oferta que determina al nivel de equilibrio de los precios interactuando con la velocidad de equilibrio y el producto potencial. De la ecuación 4 y de la ecuación cuantitativa tradicional se obtiene la expresión en logaritmos:

$$
p_{t}-p_{t}^{*}=\left(v_{t}-v_{t}^{*}\right)-\left(y_{t}-y_{t}^{*}\right)-\left(m_{t}-m_{t}\right)
$$

La ecuación 5 establece que las desviaciones del nivel de precios respecto de su nivel de equilibrio dependen de las desviaciones (o brechas) de la velocidad $(v)$ y del nivel de las transacciones $(y)$ con respecto a sus valores de equilibrio. Como la cantidad de dinero es considerada determinada siempre por la autoridad, la variable " $m$ " se elimina de la relación (5). Hallman, et al. (1991) consideran procesos de ajuste tanto para la inflación en nivel $\left(\pi_{t}=p_{t}-p_{t-1}\right)$ como en diferencias $\left(\Delta \pi_{t}=\pi_{t}-\pi_{t-1}\right)$. Dichos autores optan por esta última formulación (que implica la existencia de una raíz unitaria en la inflación). Sin embargo, en el presente estudio se encontró que para México es más apropiado el modelo en niveles. ${ }^{30}$

\footnotetext{
${ }^{30}$ Siguiendo su sugerencia, se estimó la siguiente regresión:
} 
La cuestión crucial del ejercicio es cómo computar los valores de equilibrio $y^{*}$ y $v^{*}$. Para $y^{*}$ los procedimientos típicos son utilizar una tendencia lineal o el filtro Hodrick-Prescott. ${ }^{31}$ En la investigación que dio sustento al documento se utilizó una tendencia lineal con un quiebre en 1988:01.

Para la estimación de $v^{*}$ para el lapso de 1955 a 1988 la referencia consultada fue la de Hallman et al. (1991). Dichos autores utilizaron el valor medio de la velocidad durante ese lapso mientras que para el período previo (antes de 1955) emplearon el valor ajustado de una ecuación de cointegración de la velocidad contra un conjunto de variables de "interés económico." Este último método es el que se aplicó para este documento. ${ }^{32}$ Dada la elección, la relación de este modelo con la demanda de dinero de largo plazo que se estimó antes es evidente para el caso de $m 2-p$. Para este último agregado el logaritmo de la velocidad está definido como $v=p+y-m 2$. Esta variable es no estacionaria para el caso de México (a diferencia de lo que ocurre en los Estados Unidos para el período considerado por Hallman et al. (1991)). Por tanto, es necesario encontrar alguna variable que esté cointegrada con ella.

La elección obvia para el caso de México es la tasa de interés. Estimando primero el vector de cointegración entre la velocidad y la tasa de interés se obtiene un valor para la brecha de velocidad casi idéntico al negativo del término de corrección de error para $m 2-p$. Por lo anterior, la ecuación $P^{*}$ para este último agregado resulta ser la siguiente:

$$
\pi_{t}=-\alpha_{v}\left((m 2-p)-\left(m 2-p^{*}\right)\right)_{t-1}-\alpha_{y}\left(y-y^{*}\right)_{t-1}+\sum_{i=1}^{12} \beta_{i} \pi_{t-i}+\sum_{j=1}^{12} \theta_{j} d_{j}
$$

donde $\pi_{t}$ es la inflación en el periodo $t$ y $(m 2-p)-\left(m 2-p^{*}\right)=-\left(v-v^{*}\right)$ es la brecha de dinero (en este caso casi idéntica a la brecha de la velocidad) con un rezago. La ecuación se

$$
\Delta \pi_{t}=C+\alpha_{v}\left(v-v^{*}\right)_{t-1}+\alpha_{y}\left(y-y^{*}\right)_{t-1}+\sum_{i=1}^{4} \phi_{i} \Delta \pi_{t-i}+\theta \pi_{t-1}
$$

El valor del estadístico $t$ del coeficiente de $\pi_{t-1}$ resultó igual a -5.46, lo cual, utilizando las tablas de DickeyFuller, permite rechazar confortablemente la hipótesis de raíz unitaria y, por ello, la especificación en diferencias.

${ }^{31}$ Hallman et al (1991) utilizan un estimado basado en datos preliminares del mercado de trabajo.

${ }^{32}$ En otros estudios, la velocidad de equilibrio $v^{*}$ se ha estimado a partir de una tendencia lineal, del filtro Hodrick-Prescott o de algún otro método apropiado. Por ejemplo, un método interesante introducido por 
completa con la brecha de producto y variables dicótomicas estacionales. Los valores esperados para los parámetros son $\alpha_{v}<0$ y $\alpha_{y}<0$. La versión estricta del modelo $P^{*}$ requiere además que $\alpha_{v}=\alpha_{y}$. Obsérvese que, aunque la inflación se encuentre definida en términos de alguna variable monetaria, esta ecuación no es una demanda de dinero invertida.

La ecuación 7 fue estimada para cada uno de los agregados. No obstante, solamente en el caso de $m 2-p$ fue posible identificar la brecha de dinero con el negativo de la brecha de la velocidad. ${ }^{33}$ En el resto de los agregados se utilizó como una aproximación al término de corrección de error de la sección 5.

En el Cuadro 6 se presentan los resultados de la estimación de varias ecuaciones de inflación. En la segunda columna recoge un modelo AR(1) (más variables estacionales que no se reportan). Las columnas tres a siete contienen ecuaciones basadas en el modelo $P^{*}$ para cada agregado. Estas ecuaciones son simplemente el modelo AR(1) más los respectivos términos de corrección de error y la brecha de producto. Además de las pruebas usuales de bondad de ajuste y especificación, al final del cuadro se presentan los resultados de pruebas de pronóstico estático basadas en la raíz del error cuadrático medio (ECM) y en el error de predicción absoluto medio (EPAM). Para estas pruebas el modelo se estimó hasta 1995 y se usó el resto de la muestra para evaluación.

Orphanides y Porter (1998) consiste en usar árboles de regresión para obtener una velocidad de equilibrio cambiante en el tiempo.

${ }^{33}$ Recuérdese que solamente en el caso de $m 2-p$ fue posible restringir la elasticidad ingreso a ser uno. 
Cuadro 6.

Ecuaciones de Inflación Basadas en el Modelo $P^{*}$

\begin{tabular}{|c|c|c|c|c|c|c|}
\hline Variables & \multicolumn{6}{|c|}{$\Delta \mathrm{p}$} \\
\hline$\Delta \mathrm{p}_{\mathrm{t}-1}$ & $\begin{array}{c}0.833 \\
(0.035) \\
\end{array}$ & $\begin{array}{c}0.871 \\
(0.038) \\
\end{array}$ & $\begin{array}{c}0.822 \\
(0.046)\end{array}$ & $\begin{array}{c}0.768 \\
(0.047) \\
\end{array}$ & $\begin{array}{c}0.785 \\
(0.047)\end{array}$ & $\begin{array}{c}0.889 \\
(0.035)\end{array}$ \\
\hline$\left(p^{*}-p\right)^{m y b}$ & & $\begin{array}{l}-0.013 \\
(0.007) \\
\end{array}$ & & & & \\
\hline$\left(p^{*}-p\right)^{m 1}{ }_{t-1}$ & & & $\begin{array}{l}-0.008 \\
(0.004)\end{array}$ & & & \\
\hline$\left(p^{*}-p\right)^{m 2}$ & & & & $\begin{array}{l}-0.023 \\
(0.006) \\
\end{array}$ & & \\
\hline$\left(p^{*}-p\right)^{\mathrm{m} 3}{ }_{\mathrm{t}-1}$ & & & & & $\begin{array}{c}-0.028 \\
(0.008) \\
\end{array}$ & \\
\hline$\left(p^{*}-p\right)^{m 4}{ }_{t-1}$ & & & & & & $\begin{array}{c}-0.103 \\
(0.008) \\
\end{array}$ \\
\hline $\mathrm{y}_{\mathrm{t}-1}^{\mathrm{gap}}$ & & $\begin{array}{r}0.019 \\
(0.019) \\
\end{array}$ & $\begin{array}{c}0.027 \\
(0.018) \\
\end{array}$ & $\begin{array}{c}0.040 \\
(0.018) \\
\end{array}$ & $\begin{array}{c}0.042 \\
(0.019) \\
\end{array}$ & $\begin{array}{c}0.034 \\
(0.020) \\
\end{array}$ \\
\hline $\mathrm{T}$ & 80:03-95:12 & 81:02-95:12 & \multicolumn{4}{|c|}{$80: 03-95: 12$} \\
\hline R-ajust & 0.801 & 0.808 & 0.809 & 0.818 & 0.815 & 0.805 \\
\hline $\mathrm{R}^{2}$ & 0.788 & 0.791 & 0.794 & 0.804 & 0.800 & 0.789 \\
\hline E.E. & 0.012 & 0.012 & 0.012 & 0.011 & 0.012 & 0.012 \\
\hline Asimetría & 0.989 & 0.733 & 0.841 & 0.925 & 0.842 & 0.882 \\
\hline Curtosis & 8.707 & 7.896 & 8.263 & 8.389 & 8.311 & 4.457 \\
\hline Jarque-Bera & $288.8 * *$ & $194.9 * *$ & $241.7 * *$ & $256.9 * *$ & $245.7 * *$ & $260.3 * *$ \\
\hline $\mathrm{LM}_{12} \mathrm{AR}$ & 1.031 & 1.113 & 1.235 & 1.620 & 1.567 & 1.803 \\
\hline $\mathrm{LM}_{12} \mathrm{ARCH}$ & 1.450 & 1.123 & 1.504 & 1.147 & 1.514 & 1.414 \\
\hline White-Het. & $3.9 * *$ & $3.4 * *$ & $3.3 * *$ & $2.9 * *$ & $2.9 * *$ & $3.1 * *$ \\
\hline Reset & 1.230 & 1.047 & 1.253 & 0.022 & 0.789 & 1.891 \\
\hline Predicción & \multicolumn{6}{|c|}{ 96:01-99:12 } \\
\hline ECM & 0.00647 & 0.00644 & 0.00654 & 0.00665 & 0.00658 & 0.00664 \\
\hline EPAM & 0.00544 & 0.00530 & 0.00551 & 0.00560 & 0.00554 & 0.00563 \\
\hline
\end{tabular}

*** $1 \%$ de significancia

** 5\% de significancia

* $10 \%$ de significancia 
El coeficiente del término autorregresivo resultó fuertemente significativo en todas las ecuaciones. Los términos de corrección de error fueron también significativos y tienen el signo correcto. La brecha de producto rezagada fue significativa al 5\% en las tres últimas ecuaciones. Las pruebas de especificación muestran que todas las ecuaciones tienen problemas de normalidad y heterocedasticidad de los errores.

Sin embargo, el interés se centra en las pruebas de predicción. La corrección de no normalidad por medio de variables dicótomicas empeora los resultados. Cabe observar al respecto que de acuerdo con el criterio ECM, el modelo autorregresivo simple supera a todas las demás ecuaciones, incluyendo a aquéllas en donde la brecha de producto es significativa. Con respecto al criterio EPAM, la ecuación que incluye información proveniente del agregado más básico es la única que supera al modelo autorregresivo.

En vista de los pobres resultados obtenidos mediante las ecuaciones que incluyen la brecha de dinero y la brecha de producto, se decidió probar con otras especificaciones. En el Cuadro 7 se elimina el modelo autorregresivo simple y a las otras ecuaciones se les suprimió la variable con significancia más débil (la brecha de producto) y se agregan rezagos de la tasa de crecimiento nominal del agregado respectivo. Asimismo, se reportan los mismos estadísticos de evaluación del cuadro previo. 


\section{Cuadro 7}

Modelos sin la Brecha de Producto

\begin{tabular}{|c|c|c|c|c|c|}
\hline Variables & \multicolumn{5}{|c|}{$\Delta \mathrm{p}$} \\
\hline$\Delta \mathrm{p}_{\mathrm{t}-1}$ & $\begin{array}{c}0.823 \\
(0.040) \\
\end{array}$ & $\begin{array}{c}0.776 \\
(0.047) \\
\end{array}$ & $\begin{array}{c}0.715 \\
(0.050) \\
\end{array}$ & $\begin{array}{c}0.644 \\
(0.057) \\
\end{array}$ & $\begin{array}{c}0.741 \\
(0.046) \\
\end{array}$ \\
\hline$\left(p^{*}-p\right)^{m y b}{ }_{t-1}$ & $\begin{array}{l}-0.016 \\
(0.007)\end{array}$ & & & & \\
\hline$\left(\mathrm{p}^{*}-\mathrm{p}\right)^{\mathrm{m} 1}{ }_{\mathrm{t}-1}$ & & $\begin{array}{l}-0.008 \\
(0.004) \\
\end{array}$ & & & \\
\hline$\left(p^{*}-p\right)^{m 2}{ }_{t-1}$ & & & $\begin{array}{l}-0.022 \\
(0.006) \\
\end{array}$ & & \\
\hline$\left(p^{*}-p\right)^{m 3}{ }_{t-1}$ & & & & $\begin{array}{l}-0.032 \\
(0.008)\end{array}$ & \\
\hline$\left(p^{*}-p\right)^{m 4}{ }_{t-1}$ & & & & & $\begin{array}{l}-0.016 \\
(0.007) \\
\end{array}$ \\
\hline$\Delta \mathrm{m}_{\mathrm{t}-2}$ & $\begin{array}{c}0.077 \\
(0.030) \\
\end{array}$ & & & & \\
\hline$\Delta \mathrm{m}_{\mathrm{t}-3}$ & & $\begin{array}{c}0.050 \\
(0.017)\end{array}$ & & & \\
\hline$\Delta \mathrm{m}_{\mathrm{t}-4}$ & & & & $\begin{array}{c}0.110 \\
(0.039) \\
\end{array}$ & $\begin{array}{c}0.134 \\
(0.044) \\
\end{array}$ \\
\hline$\Delta \mathrm{m}_{\mathrm{t}-5}$ & & $\begin{array}{c}0.035 \\
(0.017) \\
\end{array}$ & & & \\
\hline$\Delta \mathrm{m}_{\mathrm{t}-6}$ & & & $\begin{array}{c}0.105 \\
(0.032) \\
\end{array}$ & $\begin{array}{c}0.103 \\
(0.040) \\
\end{array}$ & $\begin{array}{r}0.122 \\
(0.045) \\
\end{array}$ \\
\hline $\mathrm{T}$ & 81:04-95:12 & $80: 07-95: 12$ & & 80:08-95:12 & \\
\hline R-ajust & 0.814 & 0.82 & 0.824 & 0.829 & 0.823 \\
\hline $\mathrm{R}^{2}$ & 0.798 & 0.804 & 0.81 & 0.814 & 0.808 \\
\hline E.E. & 0.012 & 0.012 & 0.011 & 0.011 & 0.122 \\
\hline Asimetría & 0.795 & 0.669 & 0.739 & 0.701 & 0.695 \\
\hline Curtosis & 7.28 & 7.571 & 7.066 & 6.893 & 6.754 \\
\hline Jarque-Bera & $153.73 * *$ & $175.80 * *$ & 144.26 & $131.97 * *$ & $123.52 * *$ \\
\hline $\mathrm{LM}_{12} \mathrm{AR}$ & 0.903 & 0.979 & 0.664 & 1.117 & 1.217 \\
\hline $\mathrm{LM}_{12} \mathrm{ARCH}$ & 1.526 & $1.780^{*}$ & 1.336 & $1.911 * *$ & $2.186^{* *}$ \\
\hline White-Het. & $3.265^{* *}$ & $3.066^{* *}$ & $4.257 * *$ & $6.200^{* *}$ & $6.938 * *$ \\
\hline Reset & 0.304 & 0.152 & 0.793 & 0.056 & 0.261 \\
\hline Predicción & & & :01-99:12 & & \\
\hline ECM & 0.00612 & 0.00671 & 0.00661 & 0.00583 & 0.00578 \\
\hline EPAM & 0.00514 & 0.00555 & 0.00539 & 0.00468 & 0.00463 \\
\hline
\end{tabular}


De modo inesperado, la exclusión de la brecha de producto mejora el desempeño de los modelos, en especial de los que incluyen funciones de los agregados más amplios. Este resultado puede deberse a varias razones entre las cuales destacan dos. Primero, es posible que la medida de la brecha de producto utilizada pueda ser mejorada a través de algún otro método. Con base en ello se intentó con el filtro de Hodrick-Prescot pero no mejoró el resultado reportado. Segundo, el período de evaluación es quizás demasiado corto para proporcionar conclusiones definitivas pero la contundencia de las pruebas presentadas hace dudoso que con una muestra más grande los resultados cambien sensiblemente.

Los modelos que incluyen funciones de billetes y monedas y los últimos dos agregados superan claramente al modelo autorregresivo simple. Específicamente, el error cuadrático medio y el error absoluto medio son menores para el modelo con $m 4-p$. Los resultados previos indican que los agregados monetarios proveen información útil aunque marginal para predecir la inflación. Ello, si no se cuenta con otro tipo de información.

\section{VI.2 Agregados Monetarios y Actividad Económica}

En esta última sección del trabajo se analiza la información contenida en los agregados monetarios utilizable para fines de pronóstico de la actividad económica. Esta variable se define simplemente como la tasa de crecimiento del índice de la producción industrial ( $\Delta y$ ). La ecuación general tiene la siguiente forma:

$$
\Delta y_{t}=\sum_{i=1}^{12} \Delta \phi_{i} y_{t-i}+\sum_{i=1}^{12} \eta_{i} \Delta i_{t-i}+\sum_{i=1}^{12} \Delta m_{t-i}+\sum_{i=1}^{12} \Delta P_{t-i}
$$

Esta ecuación se aplicó a cada agregado y se simplificó con base en la aplicación de pruebas de significancia para el período 1982:01-94.12 (dejándose el resto de la muestra para evaluación de pronósticos) . Los resultados se presentan en el Cuadro 8. En dicho cuadro se incluyen las pruebas de significancia, bondad de ajuste y pronóstico. En la columna dos se presenta un modelo autorregresivo puro. Las columnas cuatro, seis y ocho contienen modelos autorregresivos más rezagos de las tasas de crecimiento de los agregados $m y b, m 1, m 2$ y $m 4$ (el modelo para $m 3$ es similar al de $m 4$ ) 
Cuadro 8.

Modelos para la Actividad Industrial

\begin{tabular}{|c|c|c|c|c|c|c|c|c|}
\hline \multirow[t]{2}{*}{ Variables } & \multicolumn{8}{|c|}{$\Delta \mathrm{y}$} \\
\hline & Coef. & Inest. & Coef. & Inest. & Coef. & Inest. & Coef. & Inest. \\
\hline$\Delta \mathrm{y}_{\mathrm{t}-1}$ & -0.68 & 0.14 & -0.586 & 0.12 & 0.714 & 0.22 & -0.723 & 0.16 \\
\hline$\Delta \mathrm{y}_{\mathrm{t}-2}$ & -0.29 & 0.07 & & & -0.344 & 0.03 & -0.348 & 0.04 \\
\hline$\Delta y_{t-3}$ & & & 0.253 & 0.07 & & & & \\
\hline$\Delta y_{t-6}$ & 0.174 & 0.022 & & & & & & \\
\hline$\Delta \mathrm{myb}_{\mathrm{t}-2}$ & & & 0.120 & 0.21 & & & & \\
\hline$\Delta \mathrm{myb}_{\mathrm{t}-4}$ & & & -0.244 & 0.10 & & & & \\
\hline$\Delta \mathrm{myb}_{\mathrm{t}-11}$ & & & 0.150 & $0.56^{*}$ & & & & \\
\hline$\Delta m 1_{t-1}$ & & & & & 0.114 & 0.07 & & \\
\hline$\Delta m 1_{t-5}$ & & & & & 0.103 & 0.12 & & \\
\hline$\Delta \mathrm{m} 2_{\mathrm{t}-5}$ & & & & & & & 0.170 & 0.30 \\
\hline$\Delta \mathrm{p}_{\mathrm{t}-2}$ & & & & & -0.295 & 0.33 & -0.298 & 0.47 \\
\hline $\mathrm{T}$ & \multicolumn{8}{|c|}{ 82:01-94:12 } \\
\hline R-ajust & \multicolumn{2}{|c|}{0.644} & \multicolumn{2}{|c|}{0.667} & \multicolumn{2}{|c|}{0.665} & \multicolumn{2}{|c|}{0.659} \\
\hline $\mathrm{R}^{2}$ & \multicolumn{2}{|c|}{0.676} & \multicolumn{2}{|c|}{0.702} & \multicolumn{2}{|c|}{0.700} & \multicolumn{2}{|c|}{0.692} \\
\hline E.E. & \multirow{2}{*}{\multicolumn{2}{|c|}{$\begin{array}{c}0.025 \\
-0.049\end{array}$}} & \multicolumn{2}{|c|}{0.024} & \multicolumn{2}{|c|}{0.024} & \multicolumn{2}{|c|}{0.024} \\
\hline Asimetría & & & & & \multicolumn{2}{|c|}{-0.089} & \multicolumn{2}{|c|}{-0.126} \\
\hline Curtosis & \multicolumn{2}{|c|}{2.839} & \multicolumn{2}{|c|}{3.2} & \multirow{2}{*}{\multicolumn{2}{|c|}{$\begin{array}{l}2.695 \\
0.811\end{array}$}} & \multicolumn{2}{|c|}{2.585} \\
\hline Jarque-Bera & \multicolumn{2}{|c|}{2.231} & \multicolumn{2}{|c|}{0.655} & & & & \\
\hline $\mathrm{LM}_{12} \mathrm{AR}$ & \multicolumn{2}{|c|}{1.588} & \multicolumn{2}{|c|}{1.696} & \multicolumn{2}{|c|}{1.225} & \multicolumn{2}{|c|}{1.214} \\
\hline $\mathrm{LM}_{12} \mathrm{ARCH}$ & \multicolumn{2}{|c|}{1.465} & \multicolumn{2}{|c|}{$1.956^{*}$} & \multicolumn{2}{|c|}{1.277} & \multicolumn{2}{|c|}{1.266} \\
\hline White-Het. & & & & & 2.4 & & 2.5 & \\
\hline Reset & & & & & & & & \\
\hline Predicción & & & & $95: 0$ & $9: 12$ & & & \\
\hline ECM & & & & & 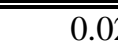 & & "0.0 & \\
\hline EPAM & & & & & & & 0.0 & \\
\hline
\end{tabular}

Los modelos se comportan satisfactoriamente en las pruebas de especificación con la excepción de la posible heterocedasticidad detectada por la prueba de White. Todos los modelos que incorpora información proveniente de los agregados monetarios superan a aquél que solamente incorpora rezagos de la propia variable dependiente. El mejor modelo de acuerdo con los criterios ECM y EPAM es el que itroduce información del agregado más básico. 


\section{Conclusiones y Comentarios Finales}

En este documento se estudian las relaciones entre los agregados monetarios (billetes y monedas y de M1 a M4), la inflación y la actividad económica en el contexto de modelos uniecuacionales. Se estimaron relaciones de largo plazo entre los distintos agregados monetarios (deflactados por el índice de precios al consumidor), la tasa de interés y el índice de la producción industrial. Los coeficientes estimados cumplen las restricciones teóricas para que las relaciones de largo plazo puedan interpretarse como demandas de dinero.

A diferencia de lo que se hacía en la literatura previa, el método de estimación utilizado (MCO-CM) permitió probar directamente las propiedades de estabilidad de cada relación de largo plazo. Las demandas más estables resultaron ser la de billetes y monedas y la de M2. Las demandas para M1 y M4 muestran posible inestabilidad de diferente origen.

Los procesos de ajuste tienen diferentes velocidades. Un desequilibrio en los billetes y monedas se elimina mucho más rápidamente que para el resto de los agregados. El modelo de corto plazo más estable y con mayor poder predictivo corresponde también al agregado más básico. La investigación mostró que las ecuaciones de demanda de corto plazo no son invertibles. Por ello, su uso para la representación del proceso que sigue alguna de las otras variables queda descartado. Siendo la no invertibilidad una implicación de la propiedad de superexogeneidad fuerte, se infiere que en México el nivel de precios determina a la cantidad de dinero y no viceversa. A pesar de lo anterior, es posible utilizar la información contenida en los agregados monetarios para hacer modelos de pronóstico para la inflación y la actividad económica. Sin embargo, la mejoría en la calidad de los pronósticos con respecto a los que produce un modelo de autorregresivo simple es modesta. 


\section{Bibliografía}

Cuthberson, K. y Luis Galindo (1999), "The Demand for Money in Mexico," The Manchester School, Vol. 67, No. 2, 154-166.

Gabriel, Vasco J,C.R., Artur C.B. da Silva López y Luis M.R.C. Nunes, (1999) “Instability in Cointegration Regressions: Further Discussion with an Application to Money Demand in Portugal," Instituto Superior de Economía, Universidad Técnica de Lisboa, Documento de Trabalho No. 3-99.

Garcés, Díaz Daniel G. (2001), “¿Cuándo es la Inflación un Fenómeno Monetario? La experiencia de México 1945-2000,”Banco de México, MIMEO.

García-Herrero, A. y Manoj Vasant Pradhan (1998), "The Domestic and Foreign Price Gap in the P-STAR Model: Evidence from Spain,” IMF Working Paper 98/64.

Hallman, J.J., Richard D. Porter and David H. Small (1991), "Is the Price Level Tied to the M2 Monetary Aggregate in the Long Run?," The American Economic Review, September, Vol. 81, No. 4, 841-858.

Hansen, B.E. (1992) “Tests for Parameter Instability in Regressions with I(1) Processes," Journal of Business and Economic Statistics, 10, 321-335.

Hendry, D. F. y Neil R. Ericsson (1991), "Modelling the demand for narrow money in the United Kingdom and the United States," European Economic Review, 35, 833-886, North-Holland.

Orphanides, A. y Richard Porter (1998), "P* Revisited:Money-Based Inflation Forecasts with a Changing EquilibriumVelocity," Board of Governors of the Federal Reserve System, May.

Phillips, P.C.B. y Bruce E. Hansen (1990), "Statistical Inference in Instrumental Variables Regression With I(1) Processes," Review of Economic Studies, 57, 99- 125.

Phillips P.C.B. y C.E. Quintos (1993), "Parameter Constancy in Cointegrating Regressions," Empirical Economics, 18, 675-706.

Vega, J. L. (1998), "Money Demand Stability: Evidence from Spain," Empirical Economics, Vol. 23. 
Lucas, R.E. (1976), Econometric policy evaluation: A critique, en Brunner, K. y Meltzer, A. (eds.), The Phillips Curve and Labor Markets, Vol. 1 of Carnegie-Rochester Conferences on Public Policy, pp. 19-46, Amsterdam: North-Holland Publishing Company.

Walsh, Carl E. (1998), "Monetary Theory and Policy," The MIT Press. 